\title{
Prédimensionnement d'un houlo-générateur pendulaire
}

\author{
M. RUELLAN*, B. ROZEL*, H. BEN AHMED*, B. MULTON*, A. BABARIT ${ }^{* *}$, A. CLEMENT ** \\ * SPEELabs/SATIE - Antenne de Bretagne (UMR CNRS 8029), ENS de Cachan - Ker Lann Campus, F35170 BRUZ \\ ${ }^{* *}$ LMF, Ecole Centrale de Nantes (UMR CNRS 6598) \\ Tel: 33 (0)2-99-05-52-64 - Fax: 33 (0)2-99-05-93-28 \\ ruellan@bretagne.ens-cachan.fr
}

\begin{abstract}
Résumé-Cet article présente le principe de prédimensionnement d'un générateur électromagnétique pour la récupération de l'énergie des vagues. Dans un premier temps, nous décrivons le principe de fonctionnement du système houlo-générateur développé dans le cadre du projet SEAREV (programme Energie du CNRS). Les couplages entre les phénomènes hydrodynamiques, mécaniques et électriques sont forts et le problème de conception du système se révèle très complexe. Il nécessite une première approche simplifiée que nous exposons ici sur un cycle de houle de durée limitée mais bien représentative de la problématique. Nous présentons une démarche d'optimisation simplifiée du système ainsi que les premiers résultats obtenus, principalement en terme de lois de commande. Une méthode d'écrêtage de la puissance est également proposée. Enfin, un prédimensionnement sur cycle d'un générateur électromagnétique à aimants permettant de satisfaire le cahier des charges est effectué avec et sans multiplicateur de vitesse mécanique.
\end{abstract}

\section{INTRODUCTION}

La houle représente une ressource énergétique renouvelable considérable (moyenne de $320 \mathrm{GW}$ en Europe ou $2800 \mathrm{TWh}$ ) [Cle_02]. En récupérer une modeste partie permettrait de contribuer significativement à la production d'énergie électrique et ne causerait qu'un faible impact sur l'environnement notamment avec de très faibles émissions de gaz à effet de serre sur cycle de vie.

Cependant la houle a la caractéristique d'être très fluctuante et non périodique. Le concept du système de récupération développé est fondé sur un balancier situé dans un flotteur fermé lui-même mis en mouvement par la houle. Le balancier ou pendule effectue des mouvements de rotation transmis à une machine électromagnétique qui récupère une partie de son énergie cinétique. Il constitue en quelque sorte un amortisseur actif et récupératif. La conception d'un générateur adapté à la houle devra avant tout tenir compte de la nature des sollicitations, notamment de leur complexité. Le dimensionnement et l'optimisation d'un tel système nécessite donc la prise en compte du couplage relativement fort existant entre les phénomènes physiques : hydrodynamiques - mécaniques - électriques (contrôle). Dans une première approche, la partie électromécanique peut être modélisée par une fonction de récupération simplifiée se limitant à un couple de récupération dont la forme, analytique ou numérique, est optimisée en vue d'obtenir, à conditions d'excitation données, une énergie électrique récupérée maximale.

Ces travaux sont menés dans le cadre du projet pluridisciplinaire SEAREV [Bab_03] impliquant le
Laboratoire de Mécanique des Fluides (LMF), le Laboratoire IRCCyN (tous deux de l'Ecole Centrale de Nantes) ainsi que le laboratoire SATIE de l'ENS de Cachan. Les deux premières entités s'intéressent plus particulièrement aux aspects hydrodynamiques et contrôle optimal. L'équipe de SATIE travaille à la conception et au dimensionnement d'un générateurrécupérateur adapté et intégré au pendule.

\section{PRINCIPE DE FONCTIONNEMENT}

Comme illustré à la figure 1 , le flotteur entièrement clos est mis en mouvement par la houle à travers des forces d'excitation. Un système mécanique s'apparentant à un pendule est placé à l'intérieur du flotteur. Le mouvement de la masse du pendule entraîne le rotor d'un générateur électromagnétique lui même couplé électriquement à une charge via un convertisseur électronique de puissance. Le contrôle de ce dernier permet d'imposer à chaque instant le couple qu'oppose le générateur au pendule.

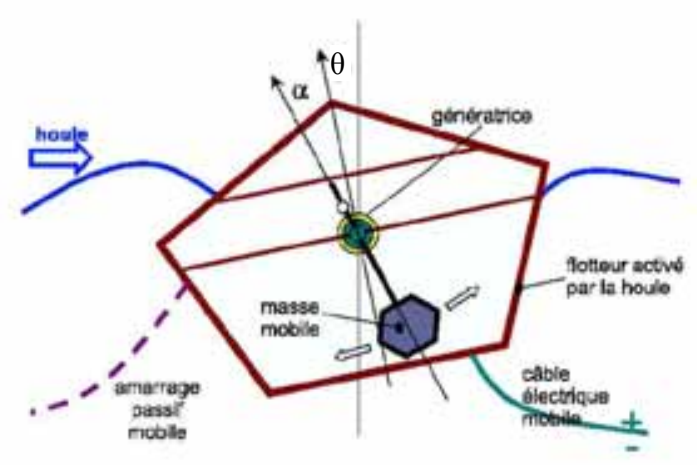

Figure 1. Schéma de principe du système houlo-générateur pendulaire

\section{MODÉLISATIONS MÉCANIQUE ET HYDRODYNAMIQUE}

La détermination de la puissance électrique récupérée nécessite le calcul des mouvements du système couplé \{flotteur + pendule\}. Ce calcul fait appel à une modélisation mécanique et hydrodynamique complexe. Nous ne décrivons ici que le principe général des modèles issus des travaux du LMF [10].

L'équation générale à résoudre est de la forme :

$$
\mathrm{M} . \ddot{\mathrm{X}}=\sum \mathrm{F}_{\mathrm{ext}}
$$


où $\mathrm{M}$ représente la matrice d'inertie du système et $\mathrm{X}=$ $\left[\mathrm{x}_{\mathrm{G}} \mathrm{z}_{\mathrm{G}} \theta \alpha\right]$ est le vecteur déplacement (cf. figure2).

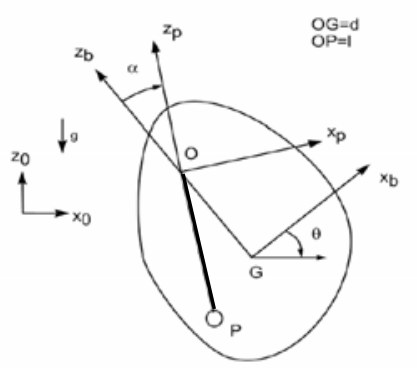

Figure 2. Repères et notations utilisés.

$\mathrm{F}_{\mathrm{ext}}$ est le vecteur de forces généralisées :

$\mathrm{F}_{\mathrm{ext}}=\mathrm{F}_{\mathrm{p}}+\mathrm{C}_{\mathrm{R}}+\mathrm{F}_{\mathrm{H}}+\mathrm{F}_{\mathrm{R}}+\mathrm{F}_{\mathrm{ex}}$.

$\mathrm{F}_{\mathrm{p}}$ représente l'effort exercé par le pendule au point $\mathrm{O}$.

Cette force est fonction de $\mathrm{X}, \dot{\mathrm{X}}$ ainsi que des paramètres géométriques du flotteur et du pendule (cf annexe A).

$\mathrm{F}_{\mathrm{H}}$ représente la force hydrostatique due à la poussée d'Archimède.

$\mathrm{F}_{\mathrm{R}}$ est la force dite de radiation correspondant à la réaction du système \{flotteur + pendule \} sur la houle.

$C_{R}$ est le couple de récupération de l'énergie exercé par le générateur électromagnétique, sa valeur est issue d'une stratégie de charge adéquate sur laquelle nous reviendrons ultérieurement.

Les forces d'excitation de la houle, $F_{\text {ex }}$ sont calculées à partir des ressources de la houle et pour une géométrie globale du flotteur imposées [10].

La houle incidente est décrite comme une superposition de plusieurs ondes élémentaires progressives, monochromatiques et dont chaque phase est aléatoire. Des études ont montré que l'état de la mer, c'est-à-dire la quantité d'énergie contenue dans chacune des ondes élémentaires qui compose une houle est une fonction lentement variable du temps. L'état de mer peut être modélisé dans l'espace des fréquences $\mathrm{f}$ par un spectre d'énergie dépendant de deux paramètres :

- un paramètre de hauteur significative (crête à creux) noté $\mathrm{H}_{1 / 3}$. Historiquement il correspond à la hauteur de la moyenne du tiers supérieur des amplitudes observées.

- une période pic $\mathrm{T}_{1}$, période possédant le plus d'énergie dans le spectre.

Les types de houle sont très variables d'un site à l'autre et en fonction de l'état de mer (de peu agitée $\mathrm{T}_{1}=5 \mathrm{~s}, \mathrm{H}_{1 / 3}=$ $0,6 \mathrm{~m}$ à grosse : $15 \mathrm{~s}-18 \mathrm{~m})$ [7].

Dans le cas du système simplifié de cette étude, seules trois composantes des efforts de la houle sur le flotteur nous intéressent : la force horizontale $\mathrm{F}_{\mathrm{ex} \_\mathrm{x}}$ (cavalement), la force verticale $\mathrm{F}_{\mathrm{ex} z}$ (pilonnement), et le moment d'axe

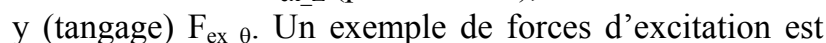
donné à la figure 3 . Dans cet exemple, la hauteur significative de la houle est de 1,5 m et sa période de pic est de $6 \mathrm{~s}$.

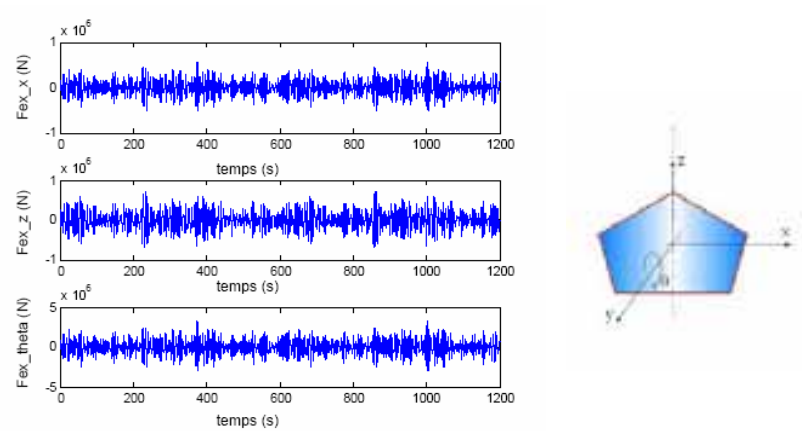

Figure 3. Exemple de forces d'excitation pour un cas particulier de houle $\left(\mathrm{H}_{1 / 3}=1.5 \mathrm{~m} ; \mathrm{T}_{1}=6 \mathrm{~s}\right)$.

Notons qu'à partir de ces considérations de houle précitées, il est possible d'exprimer un ordre de grandeur de la puissance moyenne (par unité de largeur du front) transportée par la houle. On a, dans le cas d'une houle sinusoidale [7] :

$\mathrm{P}_{\mathrm{w}}=\frac{\rho \mathrm{g}^{2}}{32 \pi} \cdot \mathrm{H}^{2} \cdot \mathrm{T}$

en $\mathrm{W} / \mathrm{m}$, où $\rho$ est la masse volumique de l'eau, g l'accélération de la pesanteur, $\mathrm{H}$ la hauteur de houle (crête à creux). Pour une mer irrégulière dont le spectre est spécifié par la hauteur significative $\mathrm{H}_{1 / 3}$ et la période caractéristique (par exemple $\mathrm{T}_{\mathrm{z}}$ la période moyenne de passage à zéro) on a (toujours en $\mathrm{W} / \mathrm{m}$ ) :

$\mathrm{P}_{\mathrm{w}}=577 \cdot \mathrm{H}_{1 / 3}^{2} \cdot \mathrm{T}_{\mathrm{z}}$

A titre d'exemple on obtient pour un état de mer peu agitée $1.0 \mathrm{~kW} / \mathrm{m}$ et $2.8 \mathrm{MW} / \mathrm{m}$ pour un état de mer extrême.

La puissance récupérable dépend ensuite de la largeur de houle captée et de l'efficacité du dispositif de conversion.

\section{Methodologie DU DIMENSIONNEMENT}

\section{A. Principe général}

Le système est soumis à une houle fluctuante et préalablement caractérisée. Afin de récupérer le maximum d'énergie, différents éléments doivent être optimisés, tout d'abord la forme hydrodynamique du flotteur, le générateur électromagnétique et enfin la stratégie de contrôle. Le couplage entre tous les éléments est fort mais on souhaite, dans une première étape, traiter le problème du dimensionnement du générateur de façon relativement découplée. Le générateur sera en effet traité comme un dispositif capable d'imposer un couple de freinage, éventuellement moteur si cela présente de l'intérêt. La réaction de ce couple de freinage sur la houle est toutefois bien prise en compte par le modèle.

A partir de ces forces d'excitation et suivant un modèle multi-physique hydrodynamique-mécanique-électrique un calcul de la puissance et de l'énergie électrique récupérée est effectué à chaque instant sur une durée fixée $\Delta \mathrm{T}\left(\Delta \mathrm{T}>>\mathrm{T}_{1}\right)$ et suffisamment longue pour que le temps de démarrage du pendule reste négligeable. Le comportement électrique du générateur (amortisseur actif du pendule) influence directement la valeur de l'énergie 
récupérée mais également, par couplage, la dynamique du système flotteur-pendule.

L'optimisation consiste à rechercher la loi de variation instantanée $\mathrm{du}$ couple électromagnétique $\mathrm{C}(\mathrm{t})$ qui maximise l'énergie récupérée. Le schéma ci-dessous illustre la méthodologie d'optimisation.

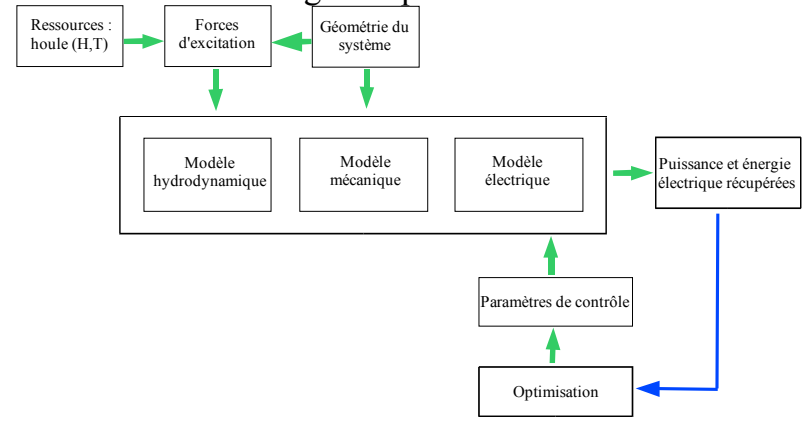

Figure 4. Synoptique de dimensionnement d'un houlo-générateur [Roz_04]

L'outil d'optimisation utilisé dans toutes les études présentées dans cet article est un algorithme évolutionnaire, le NSGA-II [4-5].

Le principe de cet algorithme repose sur le classement des individus de la population et l'application d'une stratégie élitiste. Ce classement s'opère dans l'espace des objectifs et s'appuie dans un premier temps sur la dominance au sens de Pareto. Ensuite, nous cherchons à préserver la diversité de la population.

\section{B. Optimisation sans contrainte}

Tout d'abord, nous considérons un problème sans contrainte (notamment pas d'écrêtage de puissance). Comme la recherche d'une évolution temporelle du couple exercé par le générateur constitue, dans ce contexte, un problème trop complexe à travers le nombre de variables d'optimisation que cela nécessiterait, nous avons délibérément choisi une approche progressive. Celle-ci consiste à imposer la forme générale du couple comprenant une composante de type frottement sec, frottement visqueux et raideur. Les calculs d'optimisation ont montré que seul le terme frottement visqueux était prépondérant. La forme de couple désormais considérée sera donc de type :

$$
\mathrm{C}_{\mathrm{R}}(\mathrm{t})=\beta \cdot \dot{\theta}(\mathrm{t})
$$

où $\quad \beta$ est le coefficient d'amortissement visqueux constant sur toute la durée du cycle (y compris pendant le régime transitoire de démarrage de la pendulation). Le problème d'optimisation consiste alors à rechercher les valeurs de $\beta$ telles que l'énergie récupérée $W_{\mathrm{e}}$ (donnée par (5)) soit maximale.

$$
\mathrm{W}_{\mathrm{e}}=\int_{\Delta \mathrm{T}} \beta \cdot \dot{\theta}(\mathrm{t})^{2} \cdot \mathrm{dt}
$$

Une étude paramétrique de l'influence de $\beta$ nous a permis de déterminer une valeur optimale de celui-ci (figure 5) pour un système dont les dimensions globales sont données en annexe A.

Notons ainsi que pour un coefficient d'amortissement très faible, les mouvements du pendule sont importants et donc la vitesse correspondante, cependant l'énergie récupérée est faible. Il en est de même pour $\beta$ infini où dans ce cas l'amplitude des mouvements est faible.

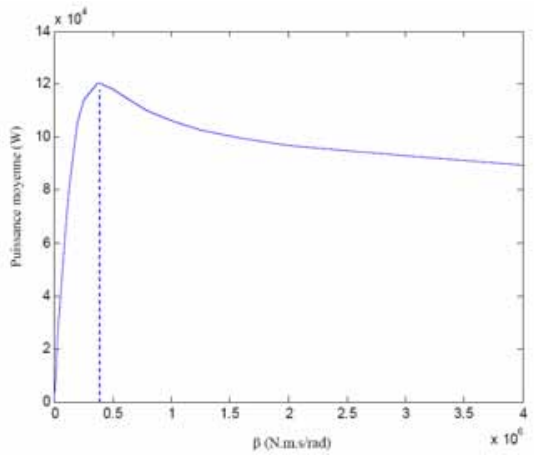

Figure 5. Exemple de l'Influence du coefficient $\beta$ sur l'énergie électrique récupérée

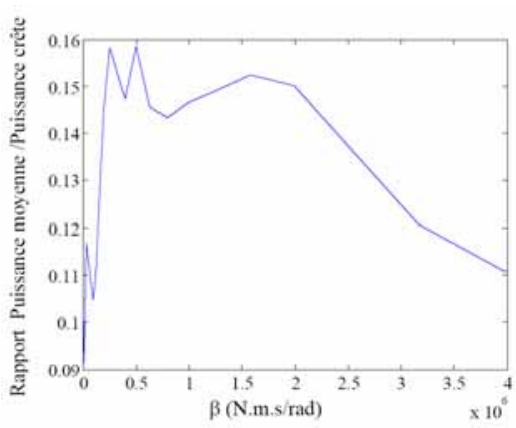

Figure 6. Exemple de l'Influence du coefficient $\beta$ sur le rapport de la puissance moyenne sur la puissance crête récupérée.

Nous observons que le rapport de la puissance moyenne sur la puissance crête est plus important (donc meilleur) pour une valeur du coefficient d'amortissement optimale.

Les figures 7 à 10 présentent les courbes de position et vitesse angulaires, couple et puissance pour un système dont le coefficient d'amortissement optimal $\beta_{\text {opt }}$ est égal à 376 kN.m.s/rad simulé avec une houle aléatoire de période pic de $6 \mathrm{~s}$ et une hauteur significative de $1.5 \mathrm{~m}$. La houle se caractérise par des ondes complexes modélisées ici , à partir d'un spectre de référence calibré par $\left(\mathrm{H}_{1 / 3}, \mathrm{~T}_{1}\right)$, comme une somme de sinusoïdes à phases aléatoires, ce qui donne un signal ou on retrouve, comme dans la nature, des « bouffées » de puissance.

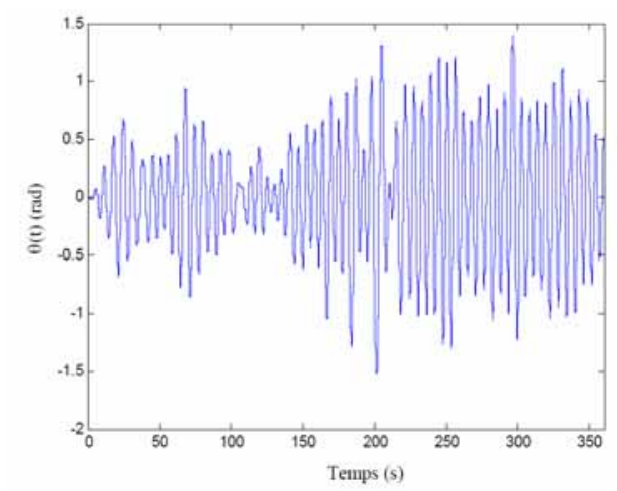

Figure 7. Position angulaire du pendule pour un amortissement optimal $\beta_{\text {opt }}$ sur cycle 


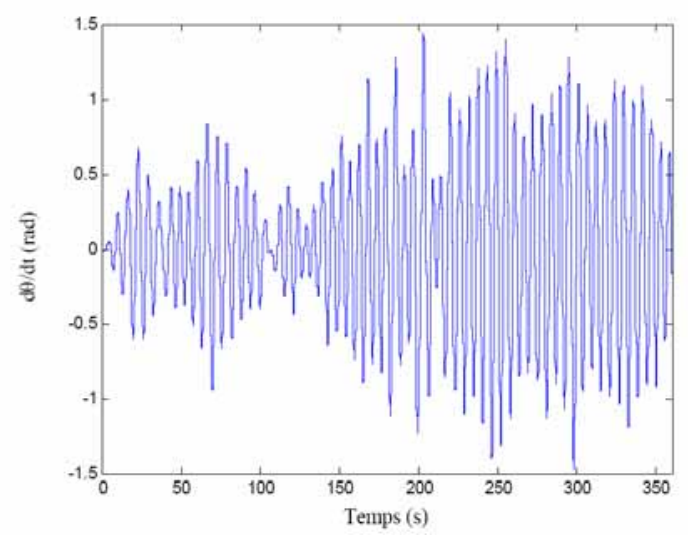

Figure 8. Vitesse angulaire du pendule pour un amortissement optimal $\beta_{\text {opt }}$ sur cycle.

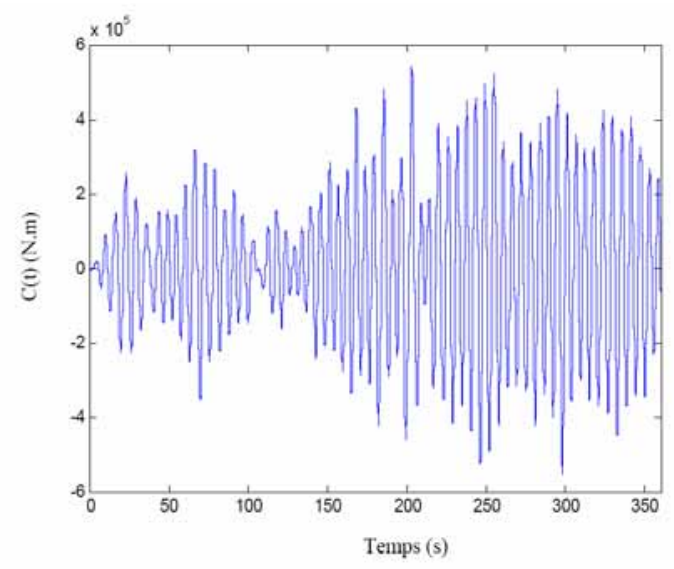

Figure 9. Couple résistant récupérateur d'énergie pour un amortissement optimal $\beta_{\text {opt }}$ sur cycle

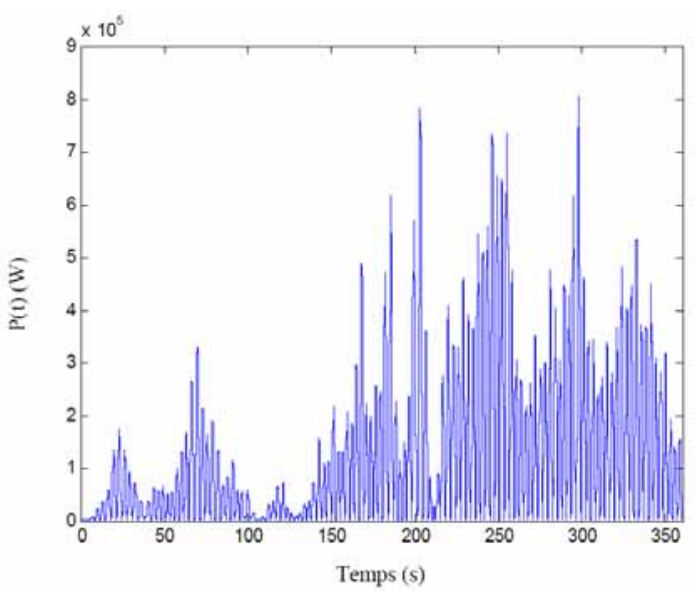

Figure 10. Puissance récupérée instantanée pour un amortissement optimal $\beta_{\text {opt }}$ sur cycle

\section{Optimisation avec contrainte d'écrêtage}

Les fluctuations importantes de la puissance récupérée engendrent un surdimensionnement du système de conversion électrique. Avec l'exemple de houle présenté précédemment (figure 10), nous obtenons une puissance moyenne de $120 \mathrm{~kW}$ (42 MJ ou $12 \mathrm{kWh}$ sur les
$360 \mathrm{~s}$ du cycle étudié) et une puissance crête de $800 \mathrm{~kW}$ pour un amortissement $\beta_{\text {opt }} \cong 376 \mathrm{kN} . \mathrm{m} . \mathrm{s} / \mathrm{rad}$. Un écrêtage de la puissance convertie (comme on peut le faire dans un système éolien) permettrait de mieux optimiser la rentabilité économique [8,9].

Cet écrêtage est obtenu dans notre cas par la modification (réduction) de la valeur du coefficient d'amortissement récupératif $\beta$. Ainsi pour les phases où la puissance est inférieure à la puissance d'écrêtage imposée, la valeur de $\beta$ est fixée à une constante (générateur fonctionnant avec un couple de type frottement visqueux) optimisée dans l'objectif de maximiser la puissance moyenne. Pour les phases où la puissance générée est supérieure à la puissance d'écrêtage, le coefficient $\beta$ varie temporellement de telle façon que la puissance générée reste égale à la puissance d'écrêtage (générateur fonctionnant à puissance constante).

Cette stratégie est illustrée par la figure 11 et par l'équation 6.
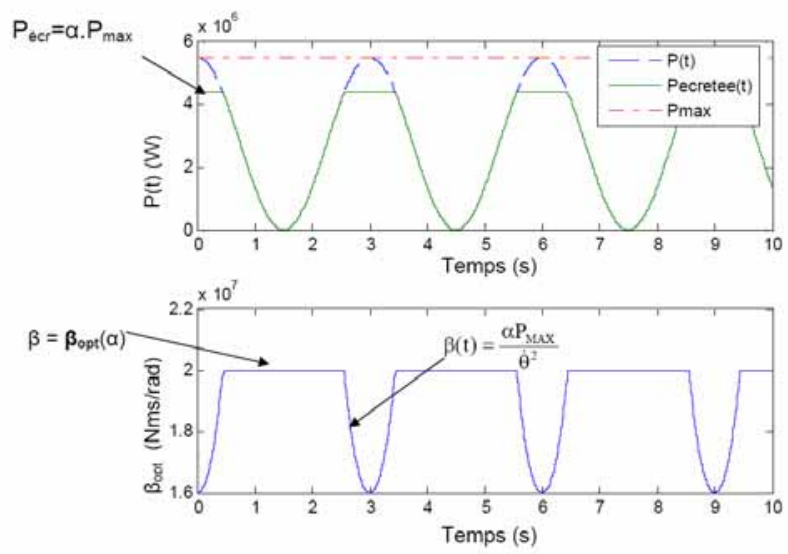

Figure 11. Principe de l'écrêtage en puissance.

$$
\beta(t)= \begin{cases}\beta_{\text {opt }} & \text { Pour } \mathrm{P}(\mathrm{t}) \leq \mathrm{P}_{\text {écr }} \\ \frac{\mathrm{P}_{\text {ecr }}}{\dot{\theta}^{2}} & \text { Pour } \mathrm{P}(\mathrm{t})>\mathrm{P}_{\text {écr }}\end{cases}
$$

Comme les mouvements du pendule sont affectés par la fonction d'écrêtage (l'amplitude des mouvements augmente), le cycle est perturbé et la valeur de l'amortissement de référence $\beta_{\mathrm{opt}}$ (dans les zones où il n'y a pas écrêtage) doit être à nouveau optimisée. Nous recherchons, par une procédure d'optimisation les valeurs optimales de $\beta$ qui maximisent la puissance moyenne (donc l'énergie récupérée) et pour différentes valeurs du taux d'écrêtage $\alpha$ tel que :

$P_{\text {écr }}=\alpha \cdot P_{\mathrm{MAX}}$.

où $\mathrm{P}_{\mathrm{MAX}}$ est la puissance crête avant écrêtage et $\mathrm{P}_{\text {écr }}$ est la puissance écrêtée.

Appliquée au même système que pour l'étude sans contrainte (annexe A) et dans les mêmes conditions à 
savoir une houle aléatoire de période $6 \mathrm{~s}$ et de hauteur significative $1.5 \mathrm{~m}$, et pour un taux d'écrêtage $\alpha$ de $80 \%$, cette optimisation a donné les résultats présentés à la figure 12 .

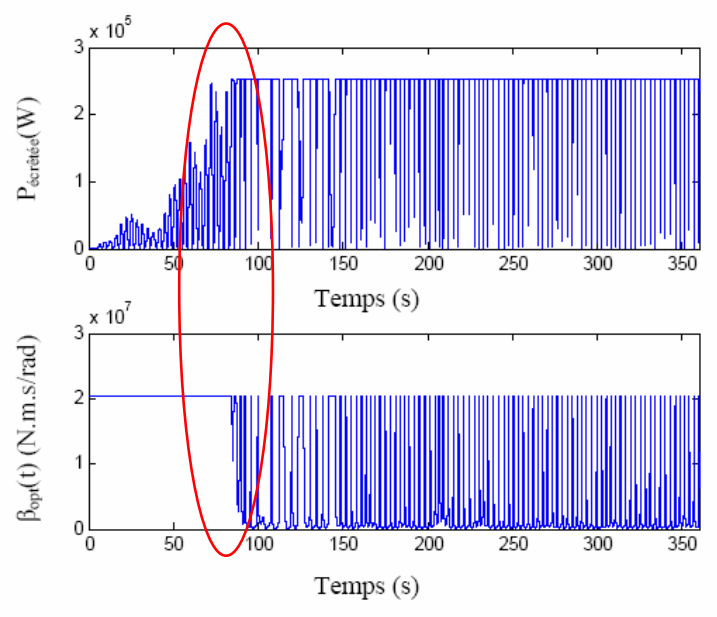

Figure 12. Allure de la puissance instantanée ainsi que de l'amortissement $\beta$ après écrêtage avec $\alpha=0.8$.
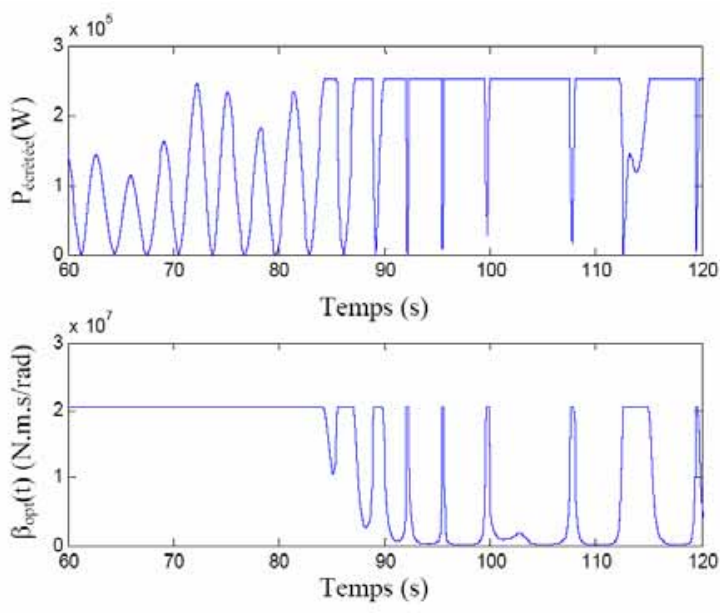

Figure 13. Zoom de la figure 12

Comme déjà souligné, l'écrêtage est réalisé en réduisant le couple électromagnétique imposé par le générateur. Durant ces phases, le pendule prend de la vitesse et l'amplitude des mouvements augmente. En effet, comme illustré figure 14, l'amplitude des mouvements est de 0.1 $\operatorname{rad}\left(5,7^{\circ}\right)$ dans le cas où l'on n'écrête pas. En situation d'écrêtage, le pendule oscille dans un premier temps autour de 0 avec une amplitude d'environ $0.1 \mathrm{rad}$. Après environ $100 \mathrm{~s}$, il effectue des oscillations avec une amplitude plus importante de $2 \mathrm{rad}$.

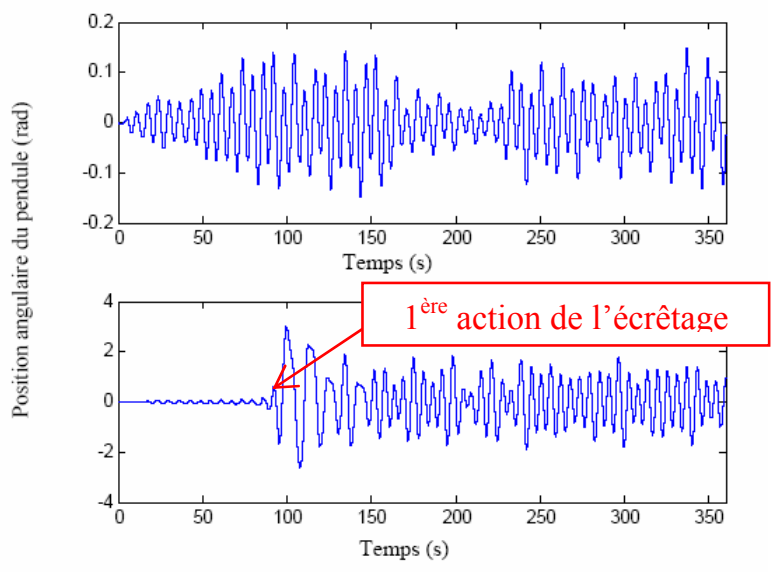

Figure 14. Position angulaire du pendule avant écrêtage (a) et après écrêtage (b) pour $\alpha=0.8$. Attention, les échelles en ordonnées ne sont pas les mêmes sur les deux figures.

La figure 15 présente deux courbes de la puissance moyenne avec amortissement optimisé selon le taux d'écrêtage (courbe bleue, croix) et sans écrêtage (courbe rouge, points) dont la valeur reste celle qui avait été optimisée (cf. figure 5). Les coefficients optimaux de $\beta$ sont donnés figure 16.

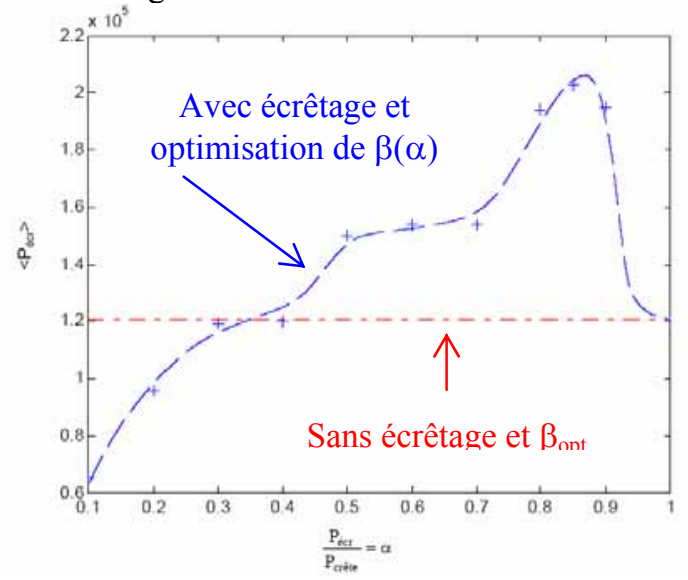

Figure 15. Puissance moyenne en mode écrêtage, en fonction du taux d'écrêtage $\alpha$.

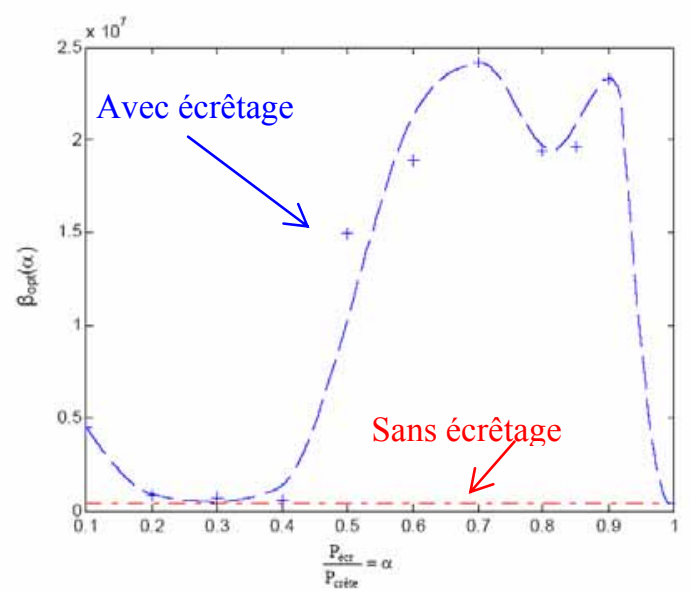

Figure 16. Evolution de $\beta_{\text {opt }}$ correspondant aux courbes de la figure 17

Ces résultats mettent en évidence l'efficacité de la stratégie d'écrêtage malgré sa simplicité ( $\beta$ constant sur 
l'ensemble du cycle en dehors des zones d'écrêtage). En effet la puissance moyenne récupérée en présence d'un écrêtage est supérieure (en particulier pour un taux d'écrêtage compris entre 0.6 et 0.9 ) à celle sans écrêtage $(\alpha=1)$, alors que l'on aurait pu s'attendre à la situation contraire, c'est-à-dire à une perte de production. On obtient en effet avec écrêtage $(\alpha=0.8)$ une puissance moyenne de $200 \mathrm{~kW}$ pour un puissance crête de $250 \mathrm{~kW}$ (donc un rapport $\mathrm{P}_{\mathrm{MAX}} /<\mathrm{P}>$ de 1.25) alors que sans écrêtage la puissance moyenne n'est que de $120 \mathrm{~kW}$ et la puissance crête de $800 \mathrm{~kW}\left(\mathrm{P}_{\mathrm{MAX}} /<\mathrm{P}>=6.7\right)$. L'écrêtage permet d'imposer des coefficients d'amortissement $\beta$ plus importants sans altérer le mouvement du pendule (cf. figure 17). Cette optimisation en puissance du dimensionnement du convertisseur électronique et des composants électriques côtés réseaux se fait au prix cependant d'une augmentation du couple résistant maximal nécessaire et donc un surdimensionnement du convertisseur électromagnétique. Nous allons dans ce qui suit étudier cet aspect.

\section{Pré-dimensionnement d'une machine synchrone adaptée à la récupération de l'énergie des vagues.}

Nous avons cherché à réaliser un pré-dimensionnement de machine sur la base des résultats, obtenus sur un cycle de houle de durée réduite pour évaluer notamment le réalisme d'un entraînement direct. Bien entendu, une étude réaliste nécessite de considérer une variété beaucoup plus importante de houles, au minimum sur une année.

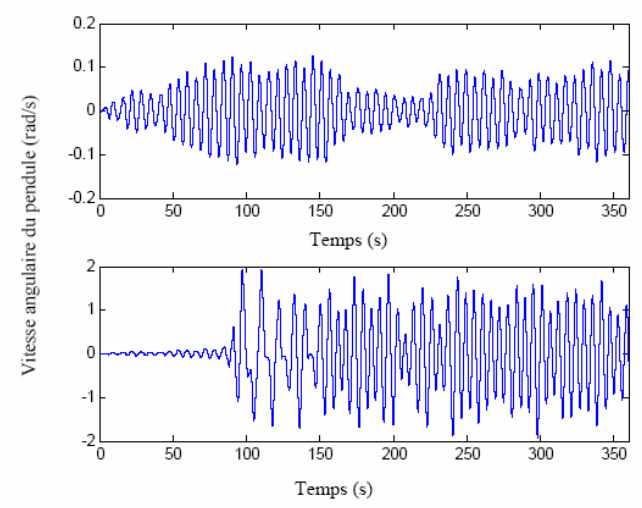

Figure 17. Vitesse angulaire du pendule sans écrêtage (a) et avec écrêtage (b) pour $\alpha=0.8$.

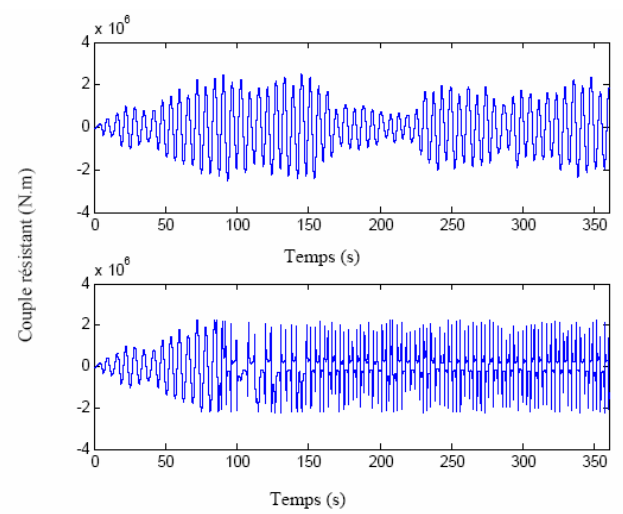

Figure 18. Couple de récupération sans écrêtage (a) et avec écrêtage (b) $(\alpha=0.8)$
Nous avons considéré deux cas : celui d'un système optimisé sans écrêtage $\left(\beta_{\mathrm{opt}}=376 \mathrm{kN} . \mathrm{m} . \mathrm{s} / \mathrm{rad}\right)$ et celui d'un système optimisé avec un écrêtage de $\alpha=0.8$ ( $\beta_{\text {opt }}=$ 20 MN.m.s/rad) pour lesquels les grandeurs principales de dimensionnement (couple et vitesse de rotation) sont données aux figures 17 et 18 .

La machine considérée pour cette pré-étude est une structure classique synchrone à aimants en surface, cylindrique à champ radial et rotor intérieur. D'autres types de machines devront être analysées et comparées dans le futur notamment discoïde à champ axial ou encore machine à bobinage global à « flux transverse ». Cependant, cette première étude permet d'affiner l'approche et les outils d'optimisation élaborés et de dégager un certain nombre de résultats qualitatifs compte tenu de la spécificité du cahier des charges considéré.

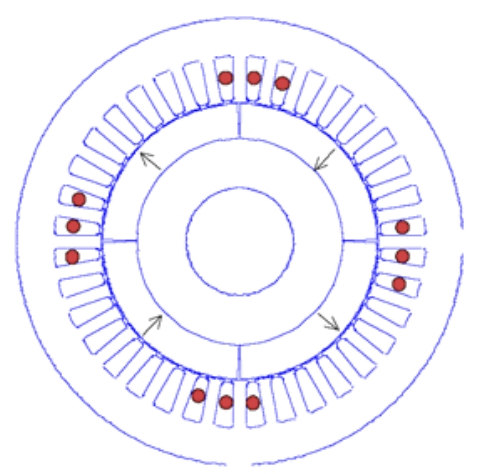

Figure 19. Architecture de principe du générateur : machine synchrone à champ radial, à couplages polaires et à aimants en surface

\section{1) Formalisme :}

Il s'agit de déterminer les caractéristiques géométriques optimales du générateur synchrone permettant de minimiser, au sens de Pareto, deux objectifs contradictoires que sont les pertes totales et le volume (ou la masse) des parties actives. Cette recherche est effectuée en considérant le couple $C_{R}(t)$ et la vitesse de rotation $\Omega(\mathrm{t})$ obtenus lors de l'optimisation système précédente sur un cycle de fonctionnement donné, ici le cycle de référence de l'article (figures 17 et 18).

Ainsi, à partir de quelques considérations simplificatrices (fer à perméabilité infinie, modèle limité au premier harmonique, refroidissement surfacique), la variation de la densité linéique efficace de courant d'induit $\mathrm{A}_{\text {Leff }}$ nécessaire pour générer le profil du couple souhaité est donnée par :

$\mathrm{A}_{\text {Leff }}(\mathrm{t})=\frac{\mathrm{C}_{\mathrm{R}}(\mathrm{t})}{\mathrm{k}}$

$\mathrm{k}$ représente la constante de couple liée au volume du rotor $\mathrm{V}_{\mathrm{r}}$ de la machine et au type d'aimants permanents utilisés :

$$
\mathrm{k}=2 \sqrt{2} \cdot \mathrm{K}_{\mathrm{B}} \cdot \mathrm{B}_{\mathrm{fm}} \mathrm{V}_{\mathrm{r}} \cos (\psi)
$$


où $\mathrm{K}_{\mathrm{B}}$ est le coefficient de bobinage, $\psi$ est l'angle d'autopilotage (déphasage entre la f.e.m. et le courant d'induit) et $\mathrm{V}_{\mathrm{r}}=\pi(\mathrm{R})^{2}$.L est la cylindrée rotorique.

L'amplitude du fondamental de l'induction d'entrefer due à l'inducteur à aimants en surface (effet de courbure négligé) peut être approchée par :

$$
\mathrm{B}_{\mathrm{fim}}=\frac{4 . \mathrm{M}}{\pi} \sin \left(\frac{\mathrm{p}}{2 \pi} \beta_{\mathrm{a}}\right) \frac{\mathrm{K}_{\mathrm{f}}}{1+\frac{\mathrm{K}_{\mathrm{c}} \mathrm{e}}{\mathrm{h}_{\mathrm{a}}} \frac{\mu_{\mathrm{a}}}{\mu_{0}}}
$$

où $\mathrm{M}$ est l'aimantation à saturation des aimants, $\beta_{\mathrm{a}}$ est l'ouverture relative des aimants $\left(\beta_{\mathrm{a}}=1\right.$ pour des aimants jointifs), $\mathrm{K}_{\mathrm{c}}$ représente le coefficient de Carter et $\mathrm{K}_{\mathrm{f}}$ est le coefficient de fuites inter-aimants [6] :

$$
\mathrm{K}_{\mathrm{f}} \cong \frac{3 \mathrm{p} \beta_{\mathrm{a}} \mathrm{K}_{\mathrm{c}} \cdot \mathrm{e}}{4 \mathrm{R}}
$$

Cette optimisation devra obéir à trois contraintes principales.

\section{a) Contrainte thermique :}

La contrainte thermique est exprimée ici en considérant un refroidissement surfacique par convection, un échauffement homogène de toute la structure et une inertie thermique suffisante pour lisser les effets des fluctuations de la puissance dissipée instantanée :

$$
\frac{\frac{1}{\Delta \mathrm{T}} \int_{\Delta \mathrm{T}}\left[\mathrm{P}_{\mathrm{J}}(\mathrm{t})+\mathrm{P}_{\mathrm{mg}}(\mathrm{t})\right] \cdot \mathrm{dt}}{\alpha_{\mathrm{th}} \cdot \mathrm{S}_{\mathrm{th}}} \leq \Delta \theta_{\text {max }}
$$

où $\mathrm{S}_{\text {th }}=2 \pi \mathrm{R}_{\text {ext }}$. L est la surface d'échange thermique correspondant à la surface externe de la machine,

$\Delta \mathrm{T}$ est la durée du cycle de référence et $\Delta \theta_{\max }$ l'échauffement global maximal.

Les vitesses de rotation étant faibles, seules les pertes Joule et magnétiques sont prises en compte. D'autre part, dans un soucis de simplification de cette première approche, un fonctionnement à flux croisé $(\psi=0)$, minimisant les pertes Joule, est considéré.

Les expressions des pertes Joule et fer instantanées sont données respectivement par (12) et (13).

$$
\mathrm{P}_{\mathrm{J}}(\mathrm{t})=\frac{8}{\pi} \rho \frac{\pi \mathrm{LRK}_{\mathrm{L}}}{\mathrm{k}_{\mathrm{r}} \mathrm{h}_{\text {enc }}\left(1+\frac{\mathrm{h}_{\text {enc }}}{2 \mathrm{R}}\right)} \cdot\left(\frac{\mathrm{C}_{\mathrm{R}}(\mathrm{t})}{\mathrm{k}}\right)^{2}
$$

avec $: K_{L} \cong 1+\left(\frac{\pi}{p} \frac{R}{L}\right)$ est un coefficient tenant compte des têtes de bobines.

$$
\begin{aligned}
\mathrm{P}_{\mathrm{mg}}(\mathrm{t})= & {\left[\mathrm{V}_{\mathrm{c}}\left(\mathrm{B}_{\text {ст }}\right)^{2}+\mathrm{V}_{\mathrm{d}}\left(2 \cdot \mathrm{B}_{\text {ет }}\right)^{2}\right] \times } \\
& \left(\frac{\mathrm{p}}{2 \pi} \Omega(\mathrm{t})\right)\left[\mathrm{k}_{\mathrm{H}}+\mathrm{k}_{\mathrm{CF}} \cdot\left(\frac{\mathrm{p}}{2 \pi} \Omega(\mathrm{t})\right)\right]
\end{aligned}
$$

$\mathrm{V}_{\text {cu }}$ représente la volume de la culasse statorique et $\mathrm{V}_{\mathrm{d}}$ le volume de la zone dentée statorique.

L'induction résultante dans la culasse magnétique est liée à celle d'entrefer par :
$\mathrm{B}_{\text {ст }}=\left(\frac{\pi}{\mathrm{p}} \frac{\mathrm{R}}{\mathrm{h}_{\mathrm{c}}}\right) \cdot \mathrm{B}_{\text {ет }}$

où : $\mathrm{B}_{\mathrm{eT}}=\sqrt{\left(\mathrm{B}_{\mathrm{fim}}\right)^{2}+\left(\mathrm{B}_{\mathrm{I}}\right)^{2}}$

représente l'induction résultante due aux champs inducteur et induit

avec $B_{I}(t) \cong \mu_{0} \sqrt{2} \frac{K_{B} \pi R}{p q\left(K_{c} e+h_{a}\right)} \cdot\left(\frac{C_{R}(t)}{k}\right)$

la composante d'induction d'entrefer due au seul champ induit.

\section{b) Contraintes magnétiques :}

Deux contraintes magnétiques sont introduites. Celles-ci sont relatives à la saturation de la culasse et à la désaimantation des aimants. Ces deux limites sont calculées en considérant l'amplitude maximale de la densité linéique de courant efficace et donc à la valeur crête du couple sur le cycle:

$\left(\mathrm{A}_{\text {Leff }}\right)_{\operatorname{MAX}}=\operatorname{MAX}\left(\frac{\mathrm{C}_{\mathrm{R}}(\mathrm{t})}{\mathrm{k}}\right)$.

$\left(\mathrm{B}_{\mathrm{cT}}\right)_{\mathrm{MAX}} \leq \mathrm{B}_{\mathrm{sat}} \quad$ : saturation

$\left(\mathrm{H}_{\mathrm{I}}\right)_{\mathrm{MAX}} \leq \mathrm{H}_{\mathrm{K}} \quad$ : limite de désaimantation à la température de fonctionnement considérée.

où : $\left(\mathrm{H}_{\mathrm{I}}\right)_{\mathrm{MAX}} \cong \sqrt{2} \frac{\mathrm{K}_{\mathrm{B}} \pi \mathrm{R}}{\mathrm{p} \cdot \mathrm{q}\left(\mathrm{K}_{\mathrm{c}} \mathrm{e}+\mathrm{h}_{\mathrm{a}}\right)} \cdot\left(\mathrm{A}_{\text {Leff }}\right)_{\mathrm{MAX}}$

2) Optimisation :

La démarche d'optimisation utilise l'algorithme d'optimisation précédent (génétique). La figure 19 présente sa structure générale.

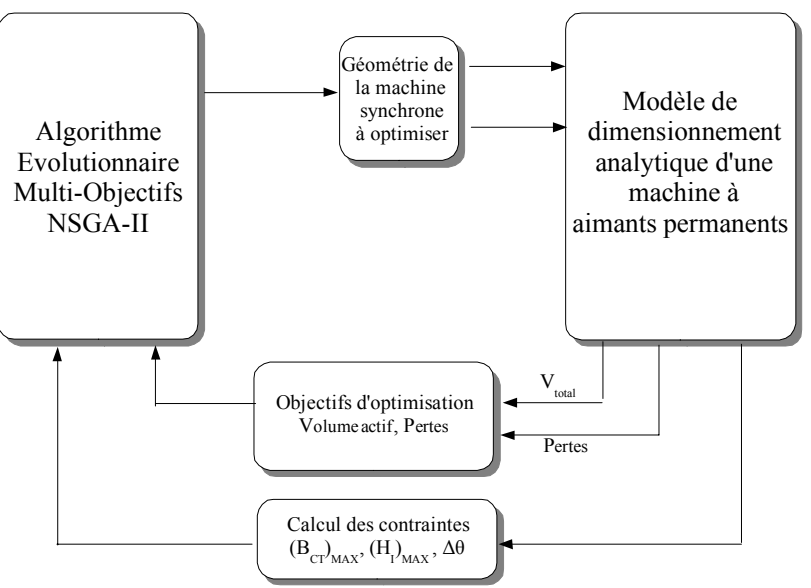

Figure 20. Synoptique général d'optimisation de la génératrice

Les objectifs retenus dans notre étude consistent à minimiser le volume des parties utiles et les pertes Joule $P_{j}$ et magnétiques $P_{m g}$ (pertes mécaniques négligées).

Les variables d'optimisation (limitées à quelques grandeurs géométriques dans cette pré-étude) sont le rayon d'alésage $R$, l'épaisseur des aimants $h_{a}$ et leur 
ouverture angulaire relative $\beta_{\mathrm{a}}$, la longueur active de la machine $\mathrm{L}$, la hauteur d'encoches $\mathrm{h}_{\mathrm{enc}}$, l'épaisseur de la culasse $h_{c u}$ (valeur identique pour les deux culasses extérieure et intérieure), le nombre de paires de pôles $\mathrm{p}$.

Nous avons fixé un certain nombre de paramètres. Ces paramètres et leurs valeurs sont donnés tableau 1 .

TABLEAU $1:$ VALEURS DES PARAMETRES CONSTANTS

\begin{tabular}{cc}
\hline Désignation & Valeurs \\
\hline Aimantation des aimants $(\mathrm{M})$ & $1 \mathrm{~T}$ \\
\hline Champ démagnétisant $\left(\mathrm{H}_{\mathrm{k}}\right)$ & $760 \mathrm{kA} / \mathrm{m}$ \\
\hline Perméabilité relative des aimants $\left(\mu_{\mathrm{ra}}\right)$ & 1 \\
\hline Induction maximale ou à saturation du fer $\left(\mathrm{B}_{\mathrm{sat}}\right)$ & $1.5 \mathrm{~T}$ \\
\hline Coefficient de pertes par hystérésis $\left(\mathrm{k}_{\mathrm{h}}\right)$ & $90 \mathrm{~A} \cdot \mathrm{m} / \mathrm{V} . \mathrm{s}$ \\
\hline Coefficient de pertes par courants de Foucault $\left(\mathrm{k}_{\mathrm{CF}}\right)$ & $1.28 \mathrm{~A} \cdot \mathrm{m} / \mathrm{V}$ \\
\hline Résistivité du cuivre à $20^{\circ} \mathrm{C}$ & $1.8 \mathrm{e}-8 \Omega . \mathrm{m}$ \\
\hline Coefficient thermique du cuivre & $0.4 \% / \mathrm{K}$ \\
\hline Coefficient de remplissage de cuivre $\mathrm{kr}$ & 0.4 \\
\hline Coefficient de bobinage $\mathrm{K}_{\mathrm{B}}$ & 0.956 \\
\hline Coefficient de Carter $\mathrm{K}_{\mathrm{c}}$ & 1.1 \\
\hline Coefficient d'échange thermique $(\alpha \mathrm{th})$ & $10 \mathrm{~W} / \mathrm{m}^{2} . \mathrm{K}$ \\
\hline Jeu mécanique $(\mathrm{e})$ & $10 \mathrm{~mm}$ \\
\hline Nombre de phases $(\mathrm{q})$ & 3 \\
\hline Angle d'autopilotage $(\psi)$ & $0{ }^{\circ}$ \\
\hline
\end{tabular}

3) Résultats :

Nous présentons dans ce qui suit les résultats obtenus pour quatre configurations :

- avec et sans écrêtage et entraînement direct. Les profils du couple et de la vitesse et du couple considérés sont celles des figures 17 et 18.

- $\quad$ avec et sans écrêtage et entraînement indirect en utilisant un multiplicateur de vitesse de rapport constant égal à 100 et de rendement constant (hypothèse simpliste) égal à $98 \%$. Dans ce cas, les profils considérés sont ceux cités précédemment augmentés (vitesse) et diminués (couple) d'un facteur 100.

Ces résultats sont présentés aux figures 21 et 22 sous la forme d'une frontière de Pareto.

Nous remarquons que la stratégie d'écrêtage, permettant d'optimiser le convertisseur électronique, entraîne un surdimensionnement du convertisseur électromagnétique tant en entraînement direct qu'indirect (figure 23) du fait de l'augmentation du couple exigé (amortissement plus fort). Cette étude reste toutefois à approfondir, nous nous sommes contentés, ici, de poser le problème.

A titre d'exemple nous présentons ci-dessous, sans et avec écrêtage ( $\alpha=80 \%)$, les dimensions de la machine (compromis entre les deux objectifs) obtenues à mêmes pertes totales pour les entraînements direct et indirect (points A et B signalés sur les figures 21 et 22).

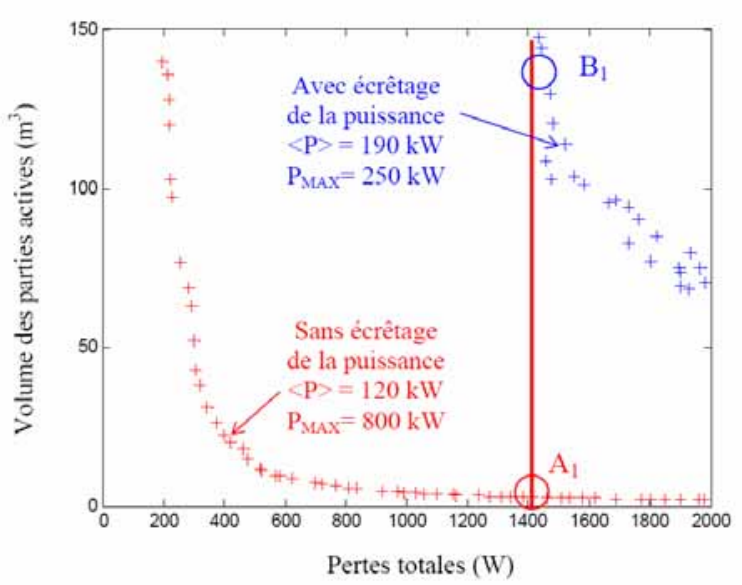

Figure 21. Optimisation en entraînement direct pour un système non écrêtée (rouge) et pour un système écrêté (bleu).

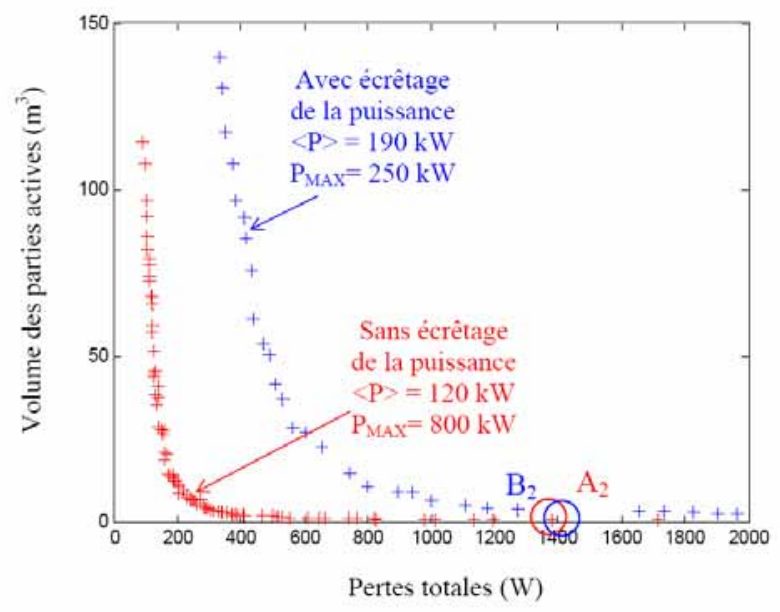

Figure 22. Optimisation en entraînement indirect (rapport $\mathrm{m}=100$ ) pour un système non écrêté (rouge) et pour un système écrêté (bleu).

TABLEAU 2 : RESULTATS D'OPTIMISATION

\begin{tabular}{ccccc}
\hline & \multicolumn{2}{c}{ Entraînement direct } & \multicolumn{2}{c}{ Entraînement indirect } \\
\hline $\begin{array}{c}\text { Masse active } \\
\text { (tonnes) }\end{array}$ & 15 & 897 & 4 & 30 \\
\hline $\begin{array}{c}\text { Masse des } \\
\text { aimants } \\
\text { (tonnes) }\end{array}$ & 3 & 90 & 0.167 & 6,2 \\
\hline $\begin{array}{c}\text { Volume actif } \\
\left.\text { (m }{ }^{3}\right)\end{array}$ & 2,7 & 144 & 0,7 & 4 \\
\hline $\begin{array}{c}\text { Masse du } \\
\text { multiplicateur } \\
\text { (tonnes) }\end{array}$ & config $\mathrm{B}_{1}$ & ${\text { config } \mathrm{A}_{2}}$ & config $\mathrm{B}_{2}$ \\
\hline $\begin{array}{c}\text { Diamètre } \\
\text { extérieur (m) }\end{array}$ & 3,6 & 10 & 2 & 2 \\
\hline $\begin{array}{c}\text { Longueur }(\mathrm{m}) \\
\text { Nombre de } \\
\text { paires de } \\
\text { pôles }\end{array}$ & 0,23 & 1,2 & 0,2 & 1,6 \\
\hline $\begin{array}{c}\mathrm{P}_{\mathrm{j}}+\mathrm{P}_{\mathrm{mg}}> \\
(\mathrm{kW})\end{array}$ & 127 & 92 & 18 & 0,6 \\
\hline
\end{tabular}




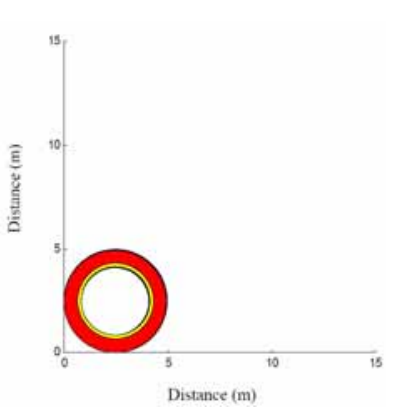

$\left(\mathrm{A}_{1}\right)$

$\left(\mathrm{A}_{2}\right)$

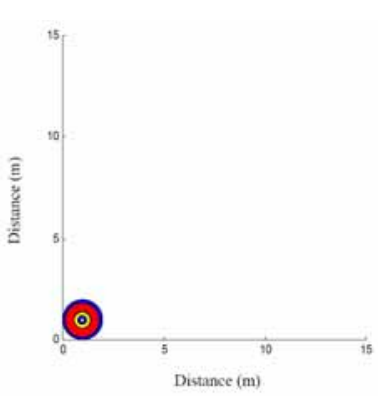

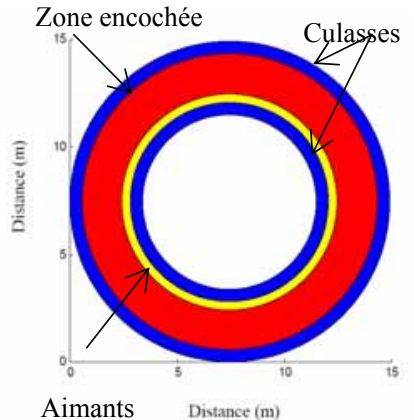

$\left(\mathrm{B}_{1}\right)$

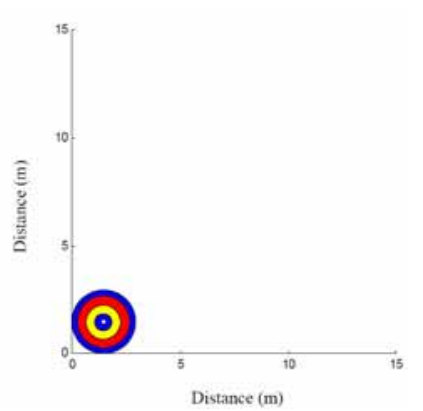

$\left(\mathrm{B}_{2}\right)$
Figure 23. Géométries optimales sans (1) et avec (2) multiplicateur et sans (A) et avec (B) écrêtage.

\section{CONCLUSION}

Cet article a montré l'ébauche de la démarche de dimensionnement d'un système houlo-générateur. La caractérisation de la houle est extrêmement complexe et l'étude s'est cantonnée à un cycle de faible durée permettant de bien poser le problème. Cela nous a permis de mettre en évidence les couplages forts que nous pressentions, notamment en situation d'écrêtage de puissance. Une optimisation sommaire (uniquement fondée sur un coefficient d'amortissement visqueux récupératif maintenu constant sur la majeure partie du cycle) du comportement du générateur-récupérateur en vue d'extraire le maximum de puissance est décrite et analysée. Les résultats montrent, dans ce contexte simplifié, qu'il existe une loi optimale d'amortissement par le générateur dont la fonction du temps reste à optimiser. En outre, nous avons montré qu'il était possible d'élaborer une stratégie d'écrêtage de la puissance électrique et qu'elle permet de réduire sensiblement les fluctuations de la puissance instantanée et donc la qualité de l'énergie produite, parfois en augmentant même l'énergie récupérée. Ceci entraîne néanmoins un surdimensionnement du convertisseur électromagnétique aussi bien en entraînement direct qu'indirect. Nous continuons à travailler sur des stratégies de contrôle améliorées, sur d'autres architectures de générateur et leur intégration au système ainsi que sur l'ajout d'une fonction de stockage d'énergie.
ANNEXE A : VALEURS DES PARAMETRES HYDRODYNAMIQUES

\begin{tabular}{|c|c|c|}
\hline Désignation & Symbole & Valeurs \\
\hline Masse du flotteur & $\mathrm{Mb}(\mathrm{kg})$ & 551000 \\
\hline Inertie du flotteur & $\mathrm{Ib}\left(\mathrm{kg} \cdot \mathrm{m}^{2}\right)$ & $0.43110^{8}$ \\
\hline Masse du pendule & $\mathrm{Mp}(\mathrm{kg})$ & 393000 \\
\hline Inertie du pendule & Ip $\left(\mathrm{kg} \cdot \mathrm{m}^{2}\right)$ & $4.6210^{6}$ \\
\hline $\begin{array}{c}\text { Distance entre le centre de } \\
\text { rotation et le centre de gravité } \\
\text { du pendule }\end{array}$ & $1(\mathrm{~m})$ & 0.9213 \\
\hline $\begin{array}{c}\text { Distance entre le centre de } \\
\text { gravité du flotteur et le centre } \\
\text { de rotation }\end{array}$ & $\mathrm{d}(\mathrm{m})$ & 0.01 \\
\hline
\end{tabular}

ANNEXE C : DEFINITIONS ET VALEURS LIMITES DES VARIABLES

\begin{tabular}{cccc}
\hline Désignation & Désignation & $\begin{array}{c}\text { Valeurs } \\
\text { Mini }\end{array}$ & $\begin{array}{c}\text { Valeurs } \\
\text { Maxi }\end{array}$ \\
\hline Épaisseur des aimants & $\mathrm{ha}(\mathrm{m})$ & 0.01 & 0.8 \\
\hline Longueur active & $\mathrm{L}(\mathrm{m})$ & 0.1 & 5 \\
\hline $\begin{array}{c}\text { Nombre de paires de } \\
\text { pôles }\end{array}$ & $\mathrm{p}$ & 1 & 150 \\
\hline Rayon d'alésage & $\mathrm{R}(\mathrm{m})$ & 0.1 & 5 \\
\hline Hauteur d'encoche $(\mathrm{m})$ & $\mathrm{h}_{\mathrm{enc}}(\mathrm{m})$ & 0.01 & 3 \\
\hline Hauteur de culasse & $\mathrm{h}_{\mathrm{cu}}(\mathrm{m})$ & 0.01 & 3 \\
\hline $\begin{array}{c}\text { Ouverture angulaire } \\
\text { relative des aimants }\end{array}$ & $\beta_{\mathrm{a}}$ & 0 & 1 \\
\hline
\end{tabular}

\section{REFERENCES}

[1] A.H. CLÉMENT et al., "Wave energy in Europe: Current status and perspectives", Renewable and Sustainable Energy Reviews, Pergamon, 6 (2002), pp.405-431.

[2] A. BABARIT, G.DUCLOS and A.H. CLÉMENT (2003): Benefit of latching control for heaving wave energy device in random sea, in: Proc. $13^{\text {th }}$ Int. Offshore and Polar Engineering Conf. ISOPE2003, Honolulu, Vol. 1, pp.341-348.

[3] B. ROZEL: «Simulation numérique d'un système houlogénérateur », Rapport de stage de magistère de l'ENS Cachan, July 2004.

[4] J. REGNIER, B.SARENI, X. ROBOAM, S. ASTIER: "Optimal design of electrical engineering systems using Pareto Genetic Algorithms", EPE 2003, Toulouse, Sept. 2003.

[5] DEB K., PRATAB A., AGRAWAL S. Heyarivau T. "A Fast and Elitist Genetic Algorithm: NSGA-II ”. IEEE Transactions on evolutionary computation, volume 6, no. 2, pp 182-197, April 2002.

[6] KONE A.D., NOGAREDE B., LAJOIE MAZENC M. : "Le dimensionnement des actionneurs électriques : un problème de programmation non linéaire. J.Phys. III (1993) 285-301.

[7] CLEMENT A.H. : "Propagation des ondes de gravité ». Cours de l'Ecole Centrale de Nantes, 2002.

[8] B. MULTON, V. DEBUSSCHERRE, G. ROBIN, O. GERGAUD, H. BEN AHMED (SATIE), A.H. CLEMENT, G. DUCLOS, A. BABARIT (LMF) « Générateur électrique combinant les ressources du soleil, du vent, de la houle et comprenant un dispositif de stockage "), rapport ADEME Convention no 0305 C 0017 (Département Energies Renouvelables), octobre 2003.

[9] A. BABARIT, H. BEN AMED, A.H. CLÉMENT, V. DEBUSSCHERE, G. DUCLOS, B. MULTON, G. ROBIN « Simulation of electricity supply of an Atlantic island by off-shore wind turbines and wave energy converters associated with a medium scale local energy storage », Proc. 8th World Renewable Energy Congress (Denver) 2004.

[10] A. BABARIT, thèse à soutenir fin 2005 au Laboratoire LMF de l'Ecole Centrale de Nantes. 